\title{
SUPERIOR VENA CAVA SYNDROME CAUSED BY PULMONARY ARTERY SARCOMA
}

John L. Faul, MD, ${ }^{\mathrm{a}}$ Tanya Wahl, MD, ${ }^{\mathrm{a}}$ Kai Ihnken, MD, ${ }^{\mathrm{b}}$ Gerald J. Berry, MD, ${ }^{\mathrm{c}}$ Ramona L. Doyle, MD, ${ }^{\mathrm{a}}$ and

Richard I. Whyte, MD, ${ }^{\mathrm{b}}$ Stanford, Calif

The obstruction of blood flow from the head, neck, and arms through the superior vena cava (SVC) results in the SVC syndrome, which can lead to life-threatening complications such as circulatory failure, venous infarcts of the brain, and laryngeal and cerebral edema. ${ }^{1}$ Most cases of SVC syndrome are due to inoperable malignant tumors, such as lung cancer $(80 \%)$ and mediastinal lymphomas (15\%). SVC syndrome caused by leiomyosarcoma of the SVC has previously been reported. ${ }^{2,3}$ This article describes a unique case of SVC syndrome caused by pulmonary artery leiomyosarcoma, a rare and highly lethal tumor. ${ }^{4}$ After treatment with radiation and chemotherapy, the tumor was successfully resected and the patient remains well 12 months later.

Clinical summary. A 51-year-old white woman was referred to this hospital for management of SVC syndrome. Fourteen months earlier she had sought treatment at another hospital for acute shortness of breath. A pulmonary angiogram at that time showed complete obstruction of blood flow in the pulmonary artery supplying the right lower lobe, and the patient received streptokinase and heparin followed by warfarin. There was no evidence of deep venous thrombosis, and neither mammography nor abdominal computed tomography (CT) indicated malignant disease. Three months later, a follow-up thoracic CT scan demonstrated a persistent filling defect in the right lower lobe pulmonary artery. Four days before admission, facial swelling and plethora developed. On the day of admission, the patient had nausea and headaches. She worked as a secretary and did not drink or smoke. Her medications included an oral contraceptive pill and warfarin.

Physical examination revealed an ill-looking woman in mild respiratory distress. Her pulse rate was 96 beats/min, blood pressure $120 / 80 \mathrm{~mm} \mathrm{Hg}$, temperature $36.5^{\circ} \mathrm{C}$, and respiratory rate 18 breaths $/ \mathrm{min}$. The face, neck, and arms were red and swollen, and veins in the chest, neck, and face were dilated. The trachea was in the midline and there was no evidence of goiter or a neck mass. The heart sounds were normal and the lungs were clear to auscultation and percussion.

From the Division of Pulmonary and Critical Care Medicine, ${ }^{\mathrm{a}}$ Department of Cardiothoracic Surgery, ${ }^{\mathrm{b}}$ and Department of Surgical Pathology, ${ }^{c}$ Stanford University Medical Center, Stanford, Calif.

Received for publication March 11, 1999; accepted for publication May 21, 1999.

Address for reprints: Richard I. Whyte, MD, Department of Cardiothoracic Surgery, Stanford University Medical Center, 300 Pasteur Dr, CVRB 205, Stanford, CA 94305-5407.

J Thorac Cardiovasc Surg 1999;118:749-50

Copyright (C) 1999 by Mosby, Inc.

0022-5223/99 $\$ 8.00+0 \quad \mathbf{1 2 / 5 4 / 1 0 0 2 8 2}$

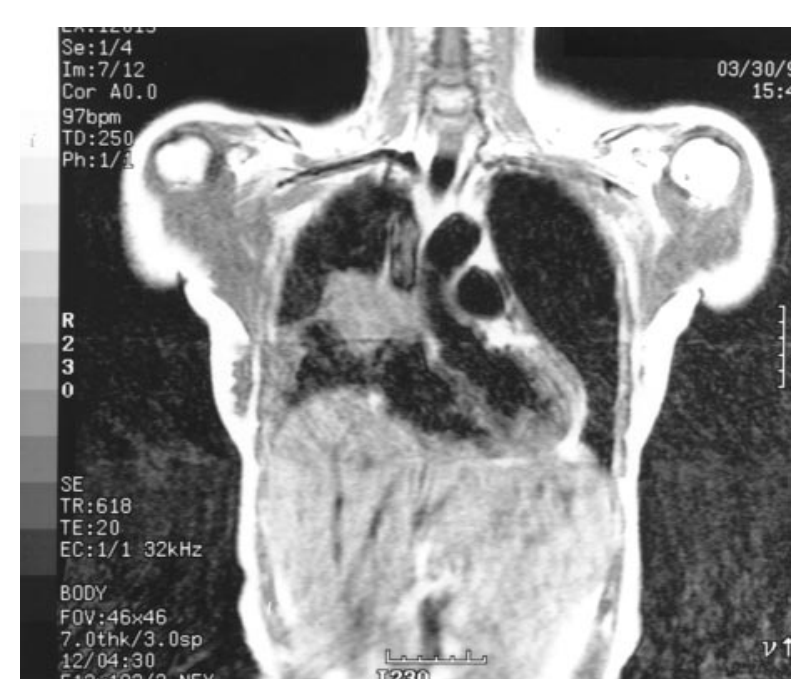

Fig 1. The patient's thoracic magnetic resonance imaging venogram, demonstrating compression of the SVC by the pulmonary artery sarcoma.

There were no abdominal masses and no leg edema. Arterial blood gas values were within normal limits. A chest radiograph demonstrated a bulky tumor in the hilum of the right lung and a small right pleural effusion. Thoracic magnetic resonance imaging revealed a large $(8 \times 5 \times 5 \mathrm{~cm})$ well-circumscribed mass at the right hilum, surrounding the bronchus intermedius, right main stem bronchus, and right lower lobe bronchus (Fig 1). The tumor arose from the right pulmonary artery, extended proximally almost to the bifurcation of the main pulmonary artery, narrowed the SVC, and indented the roof of the left atrium. Examination with a fiberoptic bronchoscope revealed extrinsic compression of the right main stem bronchus but no endobronchial lesion. CT-directed fine needle aspiration biopsy of the mass yielded pleomorphic malignant cells with prominent nucleoli and eosinophilic cytoplasmic globules. Immunohistochemical reactivity against desmin and smooth muscle actin confirmed smooth muscle differentiation, with absence of staining for epithelial, melanocytic, hematolymphoid, and vascular markers. A diagnosis of pulmonary artery leiomyosarcoma was made.

The patient underwent radiation therapy (4500 Gy) followed by 3 cycles of ifosfamide ( $2500 \mathrm{mg} /$ day for 3 days), dacarbazine (490 mg/day for 3 days), and mesna (1000 $\mathrm{mg} /$ day for 4 days). Within 3 days of treatment the symptoms of SVC syndrome had resolved, and within 4 weeks a second CT scan showed that the tumor had shrunk to half its previ- 


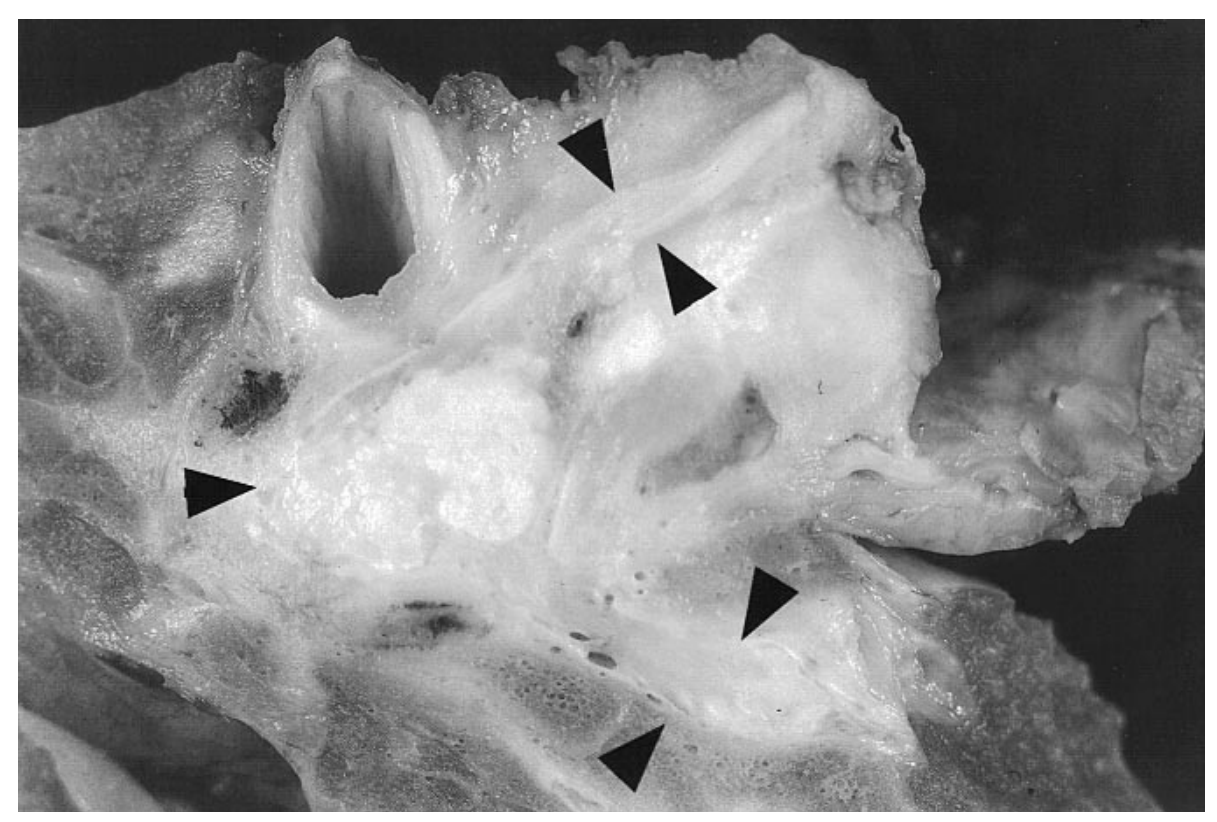

Fig 2. Coronal section of the hilum of the right lower lobe of the lung. The lumen of the pulmonary artery is filled and expanded by necrotic tumor (arrowheads).

ous size. Three months after presentation, a right pneumonectomy was performed with resection of a predominantly necrotic tumor, which involved the pulmonary artery, extended into the lung, and surrounded the right main stem bronchus (Fig 2). The tumor encased the right superior pulmonary vein, and a cuff of left atrium was removed with the lung. Cardiopulmonary bypass was available for the procedure but was not necessary. Frozen section analysis of the resection margin showed no tumor. On histologic examination of the resected specimen, only a microscopic focus of viable tumor was found. The tissues within the pulmonary artery lumen consisted of necrotic tumor, granulation tissue, fibrosis, and macrophages indicative of treated leiomyosarcoma. The patient made a full recovery from the pneumonectomy and remains well 12 months later.

Discussion. Primary pulmonary artery leiomyosarcoma is a rare and highly lethal disease. Although SVC syndrome caused by intraluminal leiomyosarcoma of the SVC has previously been reported, ${ }^{2,3}$ to our knowledge this is a unique case of SVC syndrome caused by pulmonary artery leiomyosarcoma. Several cases of leiomyosarcoma of the pulmonary artery have been described in which the clinical presentation mimics that of massive or chronic pulmonary embolism. ${ }^{1,5}$ Pulmonary artery leiomyosarcoma may lead to syncope and sudden death, and the diagnosis is therefore often made at thoracotomy or at autopsy. ${ }^{1}$ Radical surgical excision offers the only hope of cure for those with early, resectable lesions. ${ }^{5}$
Successful treatment options for SVC syndrome include thrombolysis, endovascular stent placement, radiotherapy, and transluminal angioplasty, but the choice of therapy should be directed toward treatment of the underlying cause, in addition to alleviating symptoms. ${ }^{1}$ In this case of SVC syndrome caused by advanced pulmonary artery leiomyosarcoma, combined therapy with radiation and high-dose chemotherapy led to a significant reduction in tumor bulk. The previously unresectable tumor was then removed with a standard pneumonectomy and the patient has had a good functional outcome.

\section{REFERENCES}

1. Baker GL, Barnes HJ. Superior vena cava syndrome: etiology, diagnosis, and treatment. Am J Crit Care 1992;1:54-64.

2. Weiss KS, Zidar BL, Wang S, Magovern GJ Sr, Raju RN, Lupetin $\mathrm{AR}$, et al. Radiation-induced leiomyosarcoma of the great vessels presenting as superior vena cava syndrome. Cancer 1987;60: 1238-42.

3. Tovar-Martin E, Tovar-Pardo AE, Marini M, Pimentel Y, Rois JM. Intraluminal leiomyosarcoma of the superior vena cava: a cause of superior vena cava syndrome. J Cardiovasc Surg (Torino) 1997;38:33-5.

4. Mazzucco A, Luciani GB, Bertolini P, Faggian G, Morando G, Ghimenton C. Primary leiomyosarcoma of the pulmonary artery: diagnostic and surgical implications. Ann Thorac Surg 1994;57: 222-5.

5. de Smet JM, Dieudonne P, Contu E, Le Clerc JL, Goffin Y, de Boelpape C. Pulmonary artery leiomyosarcoma treated surgically. Thorac Cardiovasc Surg 1986;34:98-9. 